\title{
Number of distinct sites visited by a subdiffusive random walker
}

\author{
Santos Bravo Yuste ${ }^{1}$, J. Klafter ${ }^{2}$, and Katja Lindenberg ${ }^{3}$ \\ (1) Departamento de Física, Universidad de Extremadura, E-06071 Badajoz, Spain \\ (2) School of Chemistry, Tel-Aviv University, Tel-Aviv, 69978 Israel \\ (3) Department of Chemistry and Biochemistry 0340, \\ and Institute for Nonlinear Science, University of California San Diego, \\ 9500 Gilman Drive, La Jolla, CA 92093-0340, USA
}

\begin{abstract}
The asymptotic mean number of distinct sites visited by a subdiffusive continuous time random walker in two dimensions seems not to have been explicitly calculated anywhere in the literature. This number has been calculated for other dimensions for only one specific asymptotic behavior of the waiting time distribution between steps. We present an explicit derivation for two cases in all integer dimensions so as to formally complete a tableaux of results. In this tableaux we include the dominant as well as subdominant contributions in all integer dimensions. Other quantities that can be calculated from the mean number of distinct sites visited are also discussed.
\end{abstract}

PACS numbers: 02.50.Ey, 05.10.Gg

The mean number of distinct sites visited by a random walker on a lattice at time $t$ after the start of the walk, $S(t)$, is a quantity often used to characterize such a walk [1, 2, 3], and many other quantities can be expressed in terms of it [4, 5]. It is of course well known that $S(t)$ depends on lattice dimension and geometry, and on the nature of the walk, that is, on the form of the waiting time distribution $\psi(t)$ between steps [4, 6, 7]. A walk is said to be subdiffusive if the asymptotic mean square displacement of the walker grows sublinearly with time, e.g., $\left\langle r^{2}(t)\right\rangle \propto t^{\gamma}$ with $0<\gamma<1$, or $\left\langle r^{2}(t)\right\rangle \propto \ln ^{\beta} t$ with $\beta>0$ [8, 9]. One way to generate such subdiffusive walks is through waiting time distributions between steps that decay sufficiently slowly to possess no finite moments. In particular,

$$
\psi(t) \sim \frac{\gamma \tau^{\gamma}}{\Gamma(1-\gamma)} t^{-1-\gamma}, \quad t \rightarrow \infty
$$

with $0<\gamma<1$ leads to [3, 10]

$$
\left\langle r^{2}(t)\right\rangle \sim \frac{\Sigma^{2}}{\Gamma(1+\gamma) \tau^{\gamma}} t^{\gamma}
$$

while

$$
\psi(t) \sim \frac{\beta}{A t[\ln (t / \tau)]^{\beta+1}}, \quad t \rightarrow \infty
$$

with $\beta>0$ leads to [8, 9]

$$
\left\langle r^{2}(t)\right\rangle \sim \Sigma^{2} A \ln ^{\beta}(t / \tau)
$$

Here $A$ is a dimensionless quantity and, in both cases, $\tau$ is a constant with units of time and $\Sigma^{2}$ is the mean square displacement of a single step. Noting the dependence of the mean square displacements on time, we will characterize the behavior of Eqs. (11) and (2) as slow and that of Eqs. (3) and (4) as ultraslow.

The Laplace transform of $\psi(t)$ will be denoted by $\psi(u)$ (recognizable by its argument). For the inverse power law distribution one has [3]

$$
\psi(u) \sim 1-(\tau u)^{\gamma}, \quad u \rightarrow 0
$$

and, for the logarithmic distribution,

$$
\psi(u) \sim 1-\frac{1}{A \ln ^{\beta}(1 / \tau u)}, \quad u \rightarrow 0 .
$$

In what follows we set $\tau$ to unity.

The probability $\chi_{n}(t)$ that the walker has taken exactly $n$ steps in time $t$ is a multiple convolution over the $\psi(t)$ most easily expressed via the relation between their Laplace transforms [6, 11],

$$
\mathcal{L} \chi_{n}(t) \equiv \chi_{n}(u)=[\psi(u)]^{n}[1-\psi(u)] / u .
$$

The mean number of distinct sites visited is given in terms of $\chi_{n}(t)$ by

$$
S(t)=\sum_{n=0}^{\infty} S_{n} \chi_{n}(t)
$$

where $S_{n}$ is the mean number of distinct sites visited by the random walker in $n$ steps. It then follows that

$$
\mathcal{L}[S(t)] \equiv S(u)=[1-\psi(u)] u^{-1} \sum_{n=0}^{\infty} S_{n}[\psi(u)]^{n}
$$

The asymptotic behavior of $S(t)$ for large $t$ can be related via the discrete Tauberian theorem [3] to the behavior of $S(u)$ as $u \rightarrow 0$, that is, to the behavior of $S(u)$ as $\psi(u) \rightarrow 1^{-}$. The discrete Tauberian theorem says that the expressions

$$
c(z)=\sum_{n=1}^{\infty} c_{n} z^{n} \sim\left(\frac{1}{1-z}\right)^{\rho} F\left(\frac{1}{1-z}\right), \quad z \rightarrow 1^{-}
$$

and

$$
c_{n} \sim \frac{n^{\rho-1}}{\Gamma(\rho)} F(n), \quad n \rightarrow \infty
$$

are equivalent if $\rho>0,\left\{c_{n}\right\}$ is a positive monotonic sequence, and $F$ is slowly varying function at infinity 
in the sense that $F(\lambda n) / F(n) \rightarrow 1$ as $n \rightarrow \infty$ for each fixed positive $\lambda$ [3]. To evaluate $S(u)$ as $u \rightarrow 0$ and use this theorem to calculate the asymptotic behavior of $S(t)$ we require the functional forms of $S_{n}$. These are well known [3] :

- For $d=1$ and $n \rightarrow \infty$

$$
S_{n}=\left(\frac{8 n}{\pi}\right)^{1 / 2}\left\{1+\frac{1}{4 n}+O\left(n^{-2}\right)\right\},
$$

- For $d=2$ and $n \rightarrow \infty$

$$
S_{n}=n L(n)
$$

with the slowly-varying function at infinity, $L(n)$, given by

$L(n)=\frac{a}{\ln (b n)}\left[1+\frac{1-\widehat{\gamma}}{\ln (b n)}+O\left(\frac{1}{\ln ^{2}(b n)}\right)\right]$

and where $\widehat{\gamma}=0.5792 \ldots$ is Euler's constant and $a$ and $b$ depend on lattice geometry. In particular, for a square lattice $a=\pi$ and $b=8$.

- For $d \geq 3$ and $n \rightarrow \infty$ the leading term is of $O(n)$ for all $d$ but the subleading terms differ,

$$
S_{n}-(1-R) n \sim \begin{cases}a \sqrt{n}, & d=3 \\ a \ln n, & d=4 \\ a, & d \geq 5\end{cases}
$$

where the probability $R$ of return to the origin depends on $d$, and where both $R$ and the constant $a$ depend on lattice geometry. In particular, for a three-dimensional simple cubic lattice $R=0.3405 \ldots[3,5]$.

Therefore, to evaluate $S(u)$ it is necessary to evaluate sums of the form [see Eq. (9)]

$$
\mathbb{S}[f ; u] \equiv \frac{1-\psi(u)}{u} \sum_{n=0}^{\infty} f(n)[\psi(u)]^{n}
$$

with $u \rightarrow 0$ and $f(n) \sim n^{\alpha}, f(n) \sim \ln n$, and $f(n) \sim$ $n L(n)$ for $n \rightarrow \infty$. Defining

$$
\phi(1 / u) \equiv \frac{1}{1-\psi(u)}
$$

and applying the discrete Tauberian theorem one finds for $u \rightarrow 0$,

$$
\begin{aligned}
\mathbb{S}\left[n^{\alpha} ; u\right] & \sim \frac{\Gamma(1+\alpha) \phi^{\alpha}}{u}, \\
\mathbb{S}[\ln n ; u] & \sim \frac{\ln \phi}{u} \\
\mathbb{S}[n L(n) ; u] & \sim \frac{\phi L(\phi)}{u} .
\end{aligned}
$$

Using these results one finds that the asymptotic dominant and first subdominant contributions to $S(u)$ for $u \rightarrow 0$ are given by:

$$
\begin{gathered}
S(u) \sim \frac{\sqrt{2}}{u}\left(\phi^{1 / 2}+\frac{1}{2} \phi^{-1 / 2}\right), \quad d=1 \\
S(u) \sim \frac{\phi}{u} L(\phi), \quad d=2
\end{gathered}
$$

and

$$
S(u) \sim(1-R) \frac{\phi}{u}+ \begin{cases}(a \sqrt{\pi} / 2 u) \phi^{1 / 2}, & d=3 \\ (a / u) \ln \phi, & d=4 \\ a / u, & d \geq 5\end{cases}
$$

To arrive at the time-dependent survival probabilities we need to apply the usual continuous Tauberian theorem 3, 12] with a specific waiting time distribution. For the inverse power law distribution (1) one has $\phi(1 / u) \sim u^{-\gamma}$ for $u \rightarrow 0$, so that the following asymptotic results for $t \rightarrow \infty$ follow:

$$
S(t) \sim \frac{\sqrt{2} t^{\gamma / 2}}{\Gamma(1+\gamma / 2)}+\frac{t^{-\gamma / 2}}{\sqrt{2} \Gamma(1-\gamma / 2)}, \quad d=1
$$

and

$$
S(t) \sim \frac{(1-R)}{\Gamma(1+\gamma)} t^{\gamma}+ \begin{cases}\frac{a \sqrt{\pi} t^{\gamma / 2}}{2 \Gamma(1+\gamma / 2)}, & d=3 \\ a \gamma \ln t, & d=4 \\ a, & d \geq 5\end{cases}
$$

We have obtained these expressions via the straightforward application of the procedure explained in [11] complemented with the discrete Tauberian theorem. The first asymptotic term for $d=1$ was obtained in [13, 14]. On the other hand, for $d=2$ we find the asymptotic (new) result

$$
\begin{aligned}
S(t) & \sim \frac{t^{\gamma}}{\Gamma(1+\gamma)} L\left(t^{\gamma}\right) \\
& \sim \frac{t^{\gamma}}{\Gamma(1+\gamma)} \frac{a}{\ln \left(b t^{\gamma}\right)}\left[1+\frac{1-\widehat{\gamma}}{\ln \left(b t^{\gamma}\right)}+O\left(\frac{1}{\ln ^{2} b t^{\gamma}}\right)\right] .
\end{aligned}
$$

In particular, the dominant contribution, used in [15], is

$$
S(t) \propto \frac{t^{\gamma}}{\ln t^{\gamma}} .
$$

For the logarithmic waiting time distribution (3) one has $\phi(1 / u) \sim A \ln ^{\beta}(1 / u)$ for $u \rightarrow 0$ so that the new asymptotic results follow:

$$
S(t) \sim \sqrt{2 A} \ln ^{\beta / 2} t+\frac{1}{\sqrt{2 A}} \ln ^{-\beta / 2} t, \quad d=1
$$




$$
\begin{aligned}
S(t) & \sim A \ln ^{\beta} t L\left(A \ln ^{\beta} t\right) \\
& \sim A \ln ^{\beta} t \frac{a}{\ln \left(b A \ln ^{\beta} t\right)}\left(1+\frac{1-\widehat{\beta}}{\ln \left(b A \ln ^{\beta} t\right)}\right) \\
& \propto \frac{\ln ^{\beta} t}{\ln \left(\ln ^{\beta} t\right)}, \quad d=2
\end{aligned}
$$

where we have exhibited the third line to highlight the dominant term, and

$$
S(t) \sim(1-R) A \ln ^{\beta} t+ \begin{cases}(a \sqrt{A \pi} / 2) \ln ^{\beta / 2} t, & d=3 \\ a \ln \left(A \ln ^{\beta} t\right), & d=4 \\ a, & d \geq 5 .\end{cases}
$$

A number of observations about these results are interesting. First, we turn to the compactness of our random walks as measured by the ratio $S(t) / V(t)$, where $V(t) \sim\left\langle r^{2}(t)\right\rangle^{d / 2}$ is the volume explored by the random walker. For the slow random walk this ratio behaves asymptotically as

$$
\frac{S(t)}{V(t)} \propto \begin{cases}t^{0}, & d=1 \\ (\ln t)^{-1}, & d=2 \\ t^{-\gamma / 2}, & d \geq 3\end{cases}
$$

For $d=1$ the walk is thus compact (the walker visits every site in the region explored). In dimensions three or greater, the walk is non-compact. In $d=2$ the logarithmic decay describes a walk that is "marginally noncompact." For the ultraslow walk we have

$$
\frac{S(t)}{V(t)} \propto \begin{cases}t^{0}, & d=1 \\ {\left[\ln \left(\ln ^{\beta} t\right)\right]^{-1},} & d=2 \\ (\ln t)^{-\beta / 2}, & d \geq 3\end{cases}
$$

While in $d=1$ the walk is again compact and in $d=2$ it is again marginally non-compact, the decay marking noncompact behavior is slower here, and remains "marginal" (logarithmic) in all dimensions above $d=2$.
A second quantity of interest that can be calculated immediately from the number of distinct sites visited is the survival probability $P(t)$ of a stationary target surrounded by a sea of moving particles of density $\rho[4,[5,6]$ :

$$
P(t)=e^{-\rho S(t)}
$$

The survival probability for the slow walk is a stretched exponential in all dimensions (with a logarithmic contribution in the exponent in two dimensions). Particularly interesting behavior is exhibited by the ultraslow walk,

$$
P(t) \sim \begin{cases}\exp \left(-c \ln ^{\beta / 2} t\right), & d=1 \\ \exp \left(-c \frac{\ln ^{\beta} t}{\ln \left(\ln ^{\beta} t\right)}\right), & d=2 \\ \exp \left(-c \ln ^{\beta} t\right), & d \geq 3,\end{cases}
$$

where $c$ is a (different) constant in each case. Note that for $d=1$ and $\beta=2$ and for $d \geq 3$ and $\beta=1$ the decay can thus be a power law, being even slower for smaller values of $\beta$. As far as we can ascertain, this is a behavior not previously observed in the target problem.

We have thus completed the panorama of "known" but previously not derived results (at least in the literature that we could locate) for the asymptotic survival probability for an inverse power law waiting time distribution (11) which leads to a slow growth of the mean square displacement. We have presented entirely new asymptotic survival probability results for the waiting time distribution (3), which leads to a growth of the mean square displacement characterized as ultraslow. We have concluded with a number of observations concerning the broader impact of these results, including the diverse range of slow decays of the survival probability in the target problem associated with the ultraslow stepping time distribution.

The research of S.B.Y. has been supported by the Ministerio de Educación y Ciencia (Spain) through grant No. FIS2007-60977 (partially financed by FEDER funds). K.L. is supported in part by the National Science Foundation under grant PHY-0354937.
[1] E. W. Montroll and G. H. Weiss, J. Math. Phys. 6, 167 (1965).

[2] G. H. Weiss, Aspects and Applications of the Random Walk (North-Holland, Amsterdam, 1994).

[3] B. H. Hughes, Random Walks and Random Environments, Volume 1: Random Walks (Clarendon Press, Oxford, 1995).

[4] A. Blumen, G. Zumofen and J. Klafter, Phys. Rev. B 30, 5379 (1984).

[5] O. Bénichou, M. Moreau and G. Oshanin, Phys. Rev. E 61, 3388 (2000).

[6] A. Blumen, J. Klafter and G. Zumofen, in Optical Spectroscopy of Glasses, ed. I. Zschokke (Reidel, Dordrecht,
1986).

[7] A. V. Barzykin and M. Tachiya, Phys. Rev. Lett. 73, 3479 (1994).

[8] S. Havlin and G. H. Weiss, J. Stat. Phys. 58, 1267 (1990).

[9] J. Dräger and J. Klafter, Phys. Rev. Lett. 84, 5998 (2000).

[10] R. Metzler and J. Klafter, Phys. Rep. 339, 1 (2000).

[11] A. Blumen, J. Klafter, B. S. White and G. Zumofen, Phys. Rev. Lett. 53, 1301 (1984).

[12] W. Feller, An Introduction to Probability Theory and its Applications Vol. 2, Second Edition (Wiley, New York, 1971).

[13] S. B. Yuste and L. Acedo, Physica A 336334 (2004). 
[14] S. B. Yuste and K. Lindenberg, Phys. Rev. E 72061103 (2005).

[15] S. B. Yuste, K. Lindenberg, J. J. Ruiz-Lorenzo, "Subdiffusion Limited Reactions" in Anomalous Transport:
Foundations and Applications R. Klages, G. Radons, I. M. Sokolov, Eds.(Wiley-VCH, Weinheim, 2007). 